\title{
Quantifying Optical Absorption of Single Plasmonic Nanoparticles and Nanoparticle Dimers Using Microstring Resonators
}

Padmanabhan Rangacharya, Varadarajan; Wu, Kaiyu; Larsen, Peter Emil; Thamdrup, Lasse Højlund Eklund; Ilchenko, Oleksii; Hwu, En Te; Rindzevicius, Tomas; Boisen, Anja

\section{Published in:}

ACS Sensors

Link to article, DOI:

10.1021/acssensors.0c00591

Publication date:

2020

Document Version

Peer reviewed version

Link back to DTU Orbit

Citation (APA):

Padmanabhan Rangacharya, V., Wu, K., Larsen, P. E., Thamdrup, L. H. E., Ilchenko, O., Hwu, E. T. Rindzevicius, T., \& Boisen, A. (2020). Quantifying Optical Absorption of Single Plasmonic Nanoparticles and Nanoparticle Dimers Using Microstring Resonators. ACS Sensors, 5(7), 2067-2075. https://doi.org/10.1021/acssensors.0c00591

\section{General rights}

Copyright and moral rights for the publications made accessible in the public portal are retained by the authors and/or other copyright owners and it is a condition of accessing publications that users recognise and abide by the legal requirements associated with these rights.

- Users may download and print one copy of any publication from the public portal for the purpose of private study or research.

- You may not further distribute the material or use it for any profit-making activity or commercial gain

- You may freely distribute the URL identifying the publication in the public portal 


\section{SENSORS}

Article

\section{Quantifying Optical Absorption of Single Plasmonic Nanoparticles and Nanoparticle Dimers Using Micro String Resonators}

Varadarajan Padmanabhan Rangacharya, Kaiyu Wu, Peter Emil Larsen, Lasse Højlund Eklund Thamdrup, Oleksii Ilchenko, En Te Hwu, Tomas Rindzevicius, and Anja Boisen

ACS Sens., Just Accepted Manuscript • DOI: 10.1021/acssensors.0c00591 • Publication Date (Web): 12 Jun 2020

Downloaded from pubs.acs.org on June 17, 2020

\section{Just Accepted}

"Just Accepted" manuscripts have been peer-reviewed and accepted for publication. They are posted online prior to technical editing, formatting for publication and author proofing. The American Chemical Society provides "Just Accepted" as a service to the research community to expedite the dissemination of scientific material as soon as possible after acceptance. "Just Accepted" manuscripts appear in full in PDF format accompanied by an HTML abstract. "Just Accepted" manuscripts have been fully peer reviewed, but should not be considered the official version of record. They are citable by the Digital Object Identifier (DOI®). "Just Accepted" is an optional service offered to authors. Therefore, the "Just Accepted" Web site may not include all articles that will be published in the journal. After a manuscript is technically edited and formatted, it will be removed from the "Just Accepted" Web site and published as an ASAP article. Note that technical editing may introduce minor changes to the manuscript text and/or graphics which could affect content, and all legal disclaimers and ethical guidelines that apply to the journal pertain. ACS cannot be held responsible for errors or consequences arising from the use of information contained in these "Just Accepted" manuscripts. 
Nanoplasmonics deals with the resonant coupling of incident photons with free electron oscillations in materials., ${ }^{1,2}$ The phenomenon is also known as surface plasmon resonance (SPR) and localized surface plasmon resonance (LSPR). ${ }^{3}$ Nanoplasmonic materials, mostly metal nanoparticles (NPs), can focus light beyond its diffraction limit. ${ }^{2,4}$ This leads to significant electromagnetic (EM) near-field enhancements and results in strong scattering and absorption of light. ${ }^{5}$ Particularly, the strong absorption gives rise to the generation of heat and its subsequent dissipation into the surrounding medium. ${ }^{4}$ Recently, nanoplasmonic heating, a.k.a. thermoplasmonics, has become a hot topic, due to its huge potential in a variety of applications, such as photothermal cancer therapy, ${ }^{6-10}$ photothermal drug delivery, ${ }^{11-13}$ photothermal imaging, ${ }^{14-16}$ and heat-assisted nanochemistry. ${ }^{17}$ In 2019, more than 300 journal articles mentioning thermoplasmonics or plasmonic heating were published according to Web of Science Core Collection. ${ }^{18}$
To facilitate applications of thermoplasmonics, there is a need to develop reliable measurement techniques to accurately measure (i) nanoscale surface temperatures and (ii) nanoscale heat sources, i.e., absorption cross sections of individual plasmonic nanostructures. It is very challenging to achieve both. Most works focus on the former. They can be divided into two categories: near-field and far-field based techniques. Near-field methods, such as scanning thermal microscopy ${ }^{19}$ are invasive, and may damage the probed nanostructures. Far-field methods, such as thermally induced refractive index change, ${ }^{20}$ surface-enhanced Raman spectroscopy (SERS), ${ }^{21}$ photoluminescence $^{22}$ and fluorescence polarization anisotropy, ${ }^{23}$ could suffer from lengthy and complex calibration processes, slow-read out rate, low-resolution, or poor temperature sensitivity. In particular, the technique that monitors the thermal-induced variation of the refractive index ${ }^{20}$ requires optically transparent samples, a liquid environment and considerable experimental and computation efforts. The SERS-based method $^{21}$ monitors the temperature dependent position of 
the $\mathrm{N} \equiv \mathrm{C}$ vibration mode of phenyl isocyanide (PIC) adsorbed on a SERS substrate made of Au. Though relatively simple, it lacks general applicability, i.e., it can only be used to probe surface temperature of SERS substrates, and has only been demonstrated on an $\mathrm{Au}$ substrate. Furthermore, obtaining reliable, temperaturedependent SERS signals from single nanoparticles or nanoparticle dimers covered by PIC would be extremely difficult due to (i) varying PIC surface coverage and (ii) low SERS signal intensities, especially when the plasmon resonance is far from the incident laser wavelength. The reported photoluminescence-based thermal sensor ${ }^{22}$ needs to be calibrated using the theoretical extinction cross section derived by employing the electric dipole approximation. This means that the sensor cannot be used to evaluate heating produced by the EM hot spots where multipoles play an important role. The method is limited to single, ellipsoidal particles with $\sim 40 \mathrm{~nm}$ in diameter or less. The fluorescence polarization anisotropy ${ }^{23}$ method offers a direct measurement of the local temperature. However, the reported fluorescence polarization anisotropy maps lack resolution to image single plasmonic particles or dimers that feature nanogaps. Recently, a method based on a customized integrating sphere has been developed to measure absorption cross sections of single plasmonic nanoparticles with submicron spatial resolution. ${ }^{24}$

Micro- and nanomechanical string resonators have lately received significant attention due to their extraordinarily high Q-factors that makes them ideal platforms for ultrasensitive sensing. ${ }^{25}$ Recently, the use of $\mathrm{Ni}, \mathrm{Al}$ and $\mathrm{SiN}$ (silicon nitride) micro- and nano string resonators as ultrasensitive temperature sensors has been explored. ${ }^{26,27}$ The developed temperature sensors exhibit a small thermal mass and a short response time. ${ }^{27}$ In addition, local temperature probing of a single plasmonic Au nanoslit and a single $\mathrm{Au}$ nanorod, has been achieved on a SiN string resonator, taking advantage of the low thermal conductivity of SiN. ${ }^{28}$ However, the method is not applicable to obtaining optical absorption cross section, which relates to efficiency of nanoscale heat generation and is thus a key parameter. Moreover, string resonators have not yet been employed to probe absorption cross sections of and temperatures near single plasmonic NPs and their dimers, which are extremely important for practical applications. ${ }^{1,7,9,29}$ The challenge mainly results from the fact that the NPs can spread across a string after sample preparation, each triggering a unique, positiondependent responsivity of the string, leading to different relative vibration frequency shifts even under identical excitation conditions.

Here, we present a first study on quantifying optical absorptions of individual plasmonic NPs and NP dimers using SiN string resonators. The probed NPs and NP dimers are composed of $\mathrm{Au}$ nanospheres (AuNS), shell- isolated $\mathrm{Au} \mathrm{NPs}, 3^{\circ} \mathrm{Ag}$ nanocubes $(\mathrm{AgNC})$ and $\mathrm{Au}$ nanostars. A superb sensitivity of $\sim 89 \mathrm{~Hz} / \mathrm{K}$ is achieved. This allows quantification of optical absorption cross sections of individual plasmonic structures, even when their plasmon resonance wavelengths are far from that of the excitation. A reliable data treatment model is developed to unravel the absorption cross sections of the probed NPs as a function of string responsivity, their positions on the string, and other parameters. The experimentally probed optical absorptions are in close agreement with those calculated via FEM simulations. Influencing factors such as polarization, surface morphology and nanogap size are discussed as well. Our study facilitates the use of plasmonic NPs to accurately control temperature at the nanoscale for future practical applications.

\section{MATERIALS AND METHODS}

Fabrication of SiN Strings: SiN micromechanical string resonators (Figure $1 \mathrm{~A}$ ) were fabricated in a cleanroom. Approximately $200 \mathrm{~nm}$ low-stress $\mathrm{SiN}$ was deposited on a double-side polished $<100>\mathrm{Si}$ wafer with a thickness of 350 $\mu \mathrm{m}$ by low-pressure chemical vapor deposition (LPCVD). The string pattern was transferred to the front side SiN using conventional UV lithography and reactive ion etching (RIE). A protective layer of SiN was deposited on top of the patterned front side by a low-frequency plasmaenhanced chemical vapor deposition (PECVD) process. Using UV lithography and RIE, a window was patterned on the back side of the wafer for subsequent release of the strings through potassium hydroxide $(\mathrm{KOH}, 28 \%)$ etch conducted at $80{ }^{\circ} \mathrm{C}$. The protective PECVD SiN layer was etched away in buffered hydrofluoric acid (1:7 BHF: DI water) at room temperature. The dimensions of the strings fabricated were $760 \times 50 \times 0.2 \mu \mathrm{m}$ (AuNS), $760 \times 15 \times 0.2 \mu \mathrm{m}$ (shell-isolated Au NPs), 1018 × 30 x $0.2 \mu \mathrm{m}$ (AuNC) and 900 x 50 x $0.2 \mu \mathrm{m}$ (Au nanostars).

Deposition of NPs onto SiN Strings: As shown in Figure 1 B, four types of NPs were deposited: i) AuNS of $\sim 200 \mathrm{~nm}$ in diameter. $\lambda_{\text {LSPR }} \approx 600 \mathrm{~nm}$ (Sigma Aldrich), ii) shellisolated Au NPs, i.e., silica-shelled AuNS, of $\sim 100 \mathrm{~nm}$ in diameter with a shell thickness of $\sim 15 \mathrm{~nm}$. $\lambda_{\text {LSPR }} \approx 585 \mathrm{~nm}$ (Nanocomposix), iii) AgNC of $\sim 100 \mathrm{~nm}$ in side length. $\lambda_{\mathrm{LSPR}}$ $\approx 565 \mathrm{~nm}$ (Nanocomposix), and iv) Au nanostars (urchins) of $\sim 100 \mathrm{~nm}$ in diameter. $\lambda_{\mathrm{LSPR}} \approx 68 \mathrm{o} \mathrm{nm}$ (Sigma Aldrich). A simple manual deposition method was adopted. A $3 \mu \mathrm{L}$ droplet of $\sim 5 \%$ solution of NPs was pipetted onto the surface of the strings. After drying, various configurations of NP clusters were obtained, including monomers, dimers, and clusters that contained multiple NPs.

SEM Characterization: A field emission Zeiss Supra 4oVP scanning electron microscope equipped with a Gemini column was used to locate and image NPs on the strings. High vacuum mode was used. The acceleration voltage was 
$10 \mathrm{kV}$. All samples were imaged directly without additional coating.

Photothermal Probing: The experimental setup is sketched in Figure ${ }_{1} C$. The chip containing the strings with the deposited NPs was firmly attached on the top of an RXY stage (SmarAct $\mathrm{GmbH}$ ). A piezoelectric actuator (Noliac A/S) was attached to the stage. This system was subsequently placed inside a vacuum chamber $\left(<10^{-5} \mathrm{mbar}\right)$. A laser-Doppler vibrometer (LDV) (MSA-50o from Polytec $\mathrm{GmbH}$ ) was used to measure the resonance frequency of the strings. The vibrometer was fitted with a 50X, $0.42 \mathrm{NA}$ objective (SEIWA Optical, Japan). A 633 nm HeNe laser was used for both probing and reading. This laser was partially polarized with an intensity ratio of 4.2:1. For photothermal imaging, a $512 \times 512$ scanning grid large enough to encompass the NP(s) was employed.

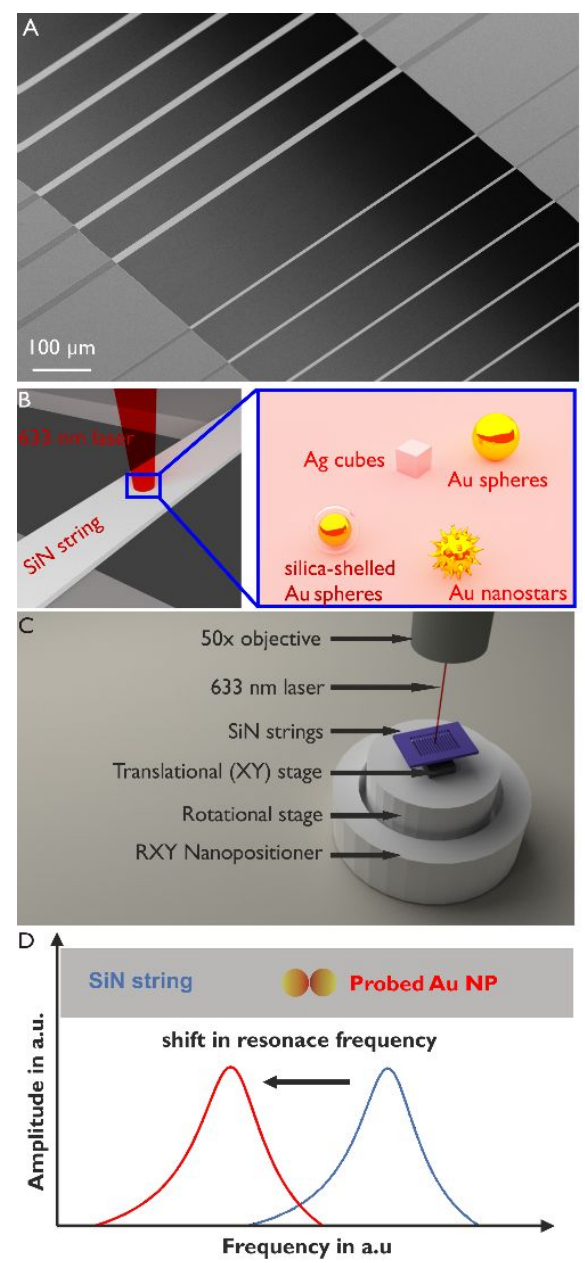

Figure 1. (A) An SEM image of the SiN micromechanical string resonators. (B) Schematic of a $633 \mathrm{~nm}$ laser illuminating a certain area on a SiN string that contains the probed NPs. (C) Schematic describing the experimental setup. A low power laser from a laser-Doppler vibrometer is used to probe NPs on a SiN string. A chip contains 25 strings. The chip is mounted on top of an RXY-stage capable of rotational and translational movements. (D) Schematic of the basic measurement principle, showing the corresponding detuning of resonance frequency of the SiN string in response to plasmonic heating of the NPs.

Measurement principle: Structurally, micromechanical string resonators are a special case of doubly clamped beams. Because of their high tensile stress, their flexural rigidity becomes negligible resulting in string-like behavior. ${ }^{31,32}$ Thus, the resonance frequency, $f_{o}$, of a micromechanical string resonator can be obtained via:27

$$
\mathrm{f}_{0}=\frac{\mathrm{n}}{2 \mathrm{~L}} \sqrt{\frac{\sigma}{\rho}}
$$

where $n$ is the resonant mode number, $L$ is the length of the string, $\sigma$ is the tensile stress and $\rho$ is the mass density of the string. In addition, the resonance frequency of a micromechanical resonator is a function of temperature. The localized temperature dependent resonance frequency $f(T)$ of the string is given by:

$$
\mathrm{f}(\mathrm{T})=\frac{\mathrm{n}}{2 \mathrm{~L}} \sqrt{\frac{\sigma-E \alpha_{\mathrm{str}} \Delta T}{\rho}}
$$

Here, $E$ is the Young's Modulus of the string material, $\alpha_{s t r}$ is the thermal expansion coefficient of the string and $\Delta T$ is the change in temperature. Temperature induced changes in $E, \rho$ and $L$ can be neglected in this model. ${ }^{26,27}$ The difference in resonance frequency (Figure $1 \mathrm{D}$ ) can be attributed to the heat generated by the the plasmonic $N P(s)$. The relative shift in frequency $\delta f$ in a string is given by:

$$
\delta f=\frac{f(T)-f_{0}}{f_{0}}
$$

Substituting equation (3) with (1) and (2), we arrive at equation (4), which calculates the change in temperature $\Delta T$ as a function of the relative frequency shift $\delta f$, which is obtained experimentally:

$$
\Delta T=2 \frac{\rho\left(2 L f_{0}\right)^{2}}{E \alpha_{s t r}}\left[1-(\delta f+1)^{2}\right]
$$

Data analysis: The photothermal imaging of the NPs is performed by scanning the string surface using an LDV, shown in Figure $2 \mathrm{~A}$. The subsequent data analysis is illustrated in Figure 2B-H. Specifically, first, the measured resonance frequency values at each point are fitted using a quasi-Lorentzian fit (Figure $2 \mathrm{~B}$ ), as the amplitude response of the string resonator can be approximated by a quasiLorentzian function. 33 Then, baseline correction is performed (Figure $2 \mathrm{C}$ ). Small sources of noise such as frequency drift over time or non-perfect angular alignment are removed by fitting a plane to the spatially distributed resonance frequency measurements. 


\section{A LDV measurement grid}

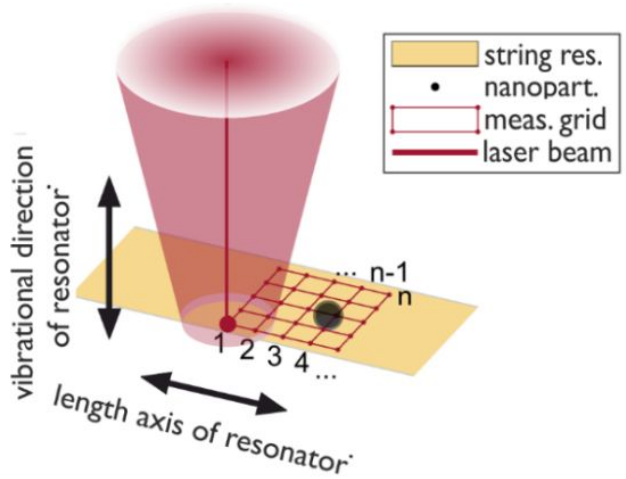

B quasi-Lorentzian fitting

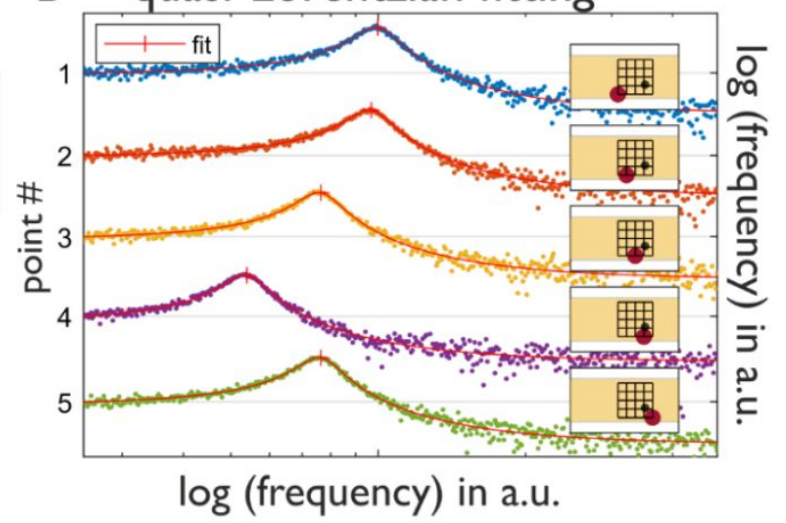

\section{C baseline correction $D$ outlier detection $E$ starting point for fit}

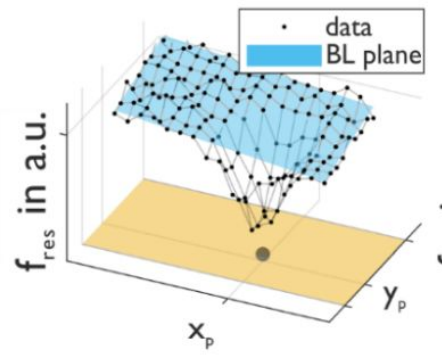

F 2D Gaussian fit

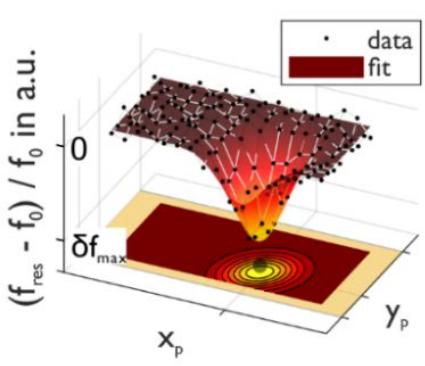

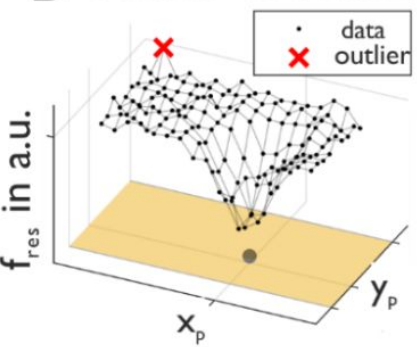

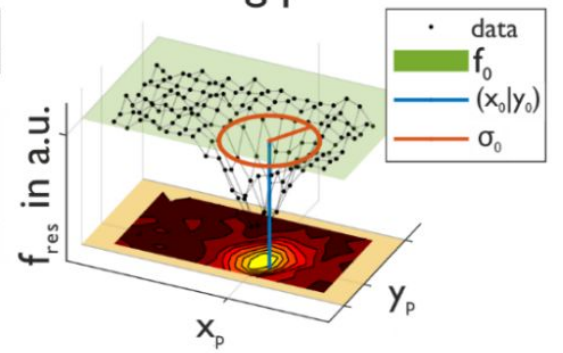

G
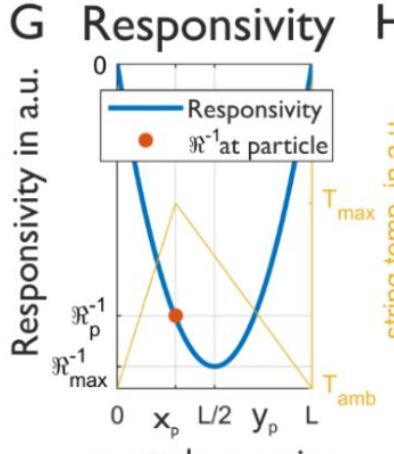

partıcle position
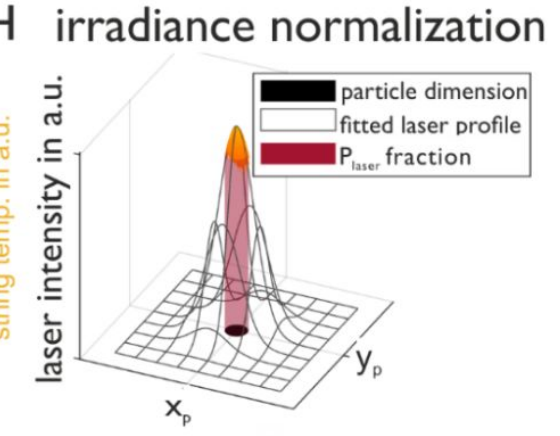

Figure 2. (A) An LDV is used to probe the NP(s). A scanning grid is first created to encompass the NP(s). Afterwards, the resonance frequency is measured at each point on the grid. (B-H) Detailed data analysis steps. (B) The measured resonance frequency values at each point are fitted using a quasi-Lorentzian fit. (C, D) Baseline correction and outlier elimination followed by determination of the resonance frequency $f_{o}$ of the string resonator. (E) A starting point for a Gaussian fit is defined by identifying the peak in the map. (F) A photothermal image represented by the relative frequency shift $\delta \mathrm{f}$ is subsequently obtained after a $2 \mathrm{D}$ Gaussian fit. $(\mathrm{G})$ The effects of location of $\mathrm{NP}(\mathrm{s})$ on the string are removed according to responsivity of the string. (H) Irradiance normalization is performed to estimate the fraction of laser power directed at the NP(s) contributing to the frequency shift.

Afterwards, outliers introduced by dirt or other imperfections on the string or in the optical path of the LDV laser are removed (Figure 2D). Subsequently, a starting point for a Gaussian fit is set by picking the center of the absorption peak with a diameter (Figure 2E,F). The diameter corresponds to FWHM of the $2 \mathrm{D}$ intensity distribution of the readout laser beam. Next, the location effect due to responsivity of the string (Figure $2 \mathrm{G}$ ) is normalized. The responsivity $(\Re)$ of a string resonator to a point source of power directed at its center is given by:33

$$
\mathfrak{R}=-\frac{1 E L{ }^{\alpha}{ }_{\text {str }}}{16 \kappa \mathrm{A} \sigma} \mathrm{f}_{0}
$$

where $A$ is the cross-sectional area of the string resonator and $\kappa$ is the thermal conductivity of the string material. Modifying equation (5) suitably for the case when the point source of power (and hence, heat) is directed at any arbitrary point ' $x$ ' along a string of length $L$, we can compute responsivity $(\mathfrak{R})$ of the absorbed power at ' $x$ ' as: 
Equation (6) clearly indicates that the responsivity of the string varies across the length of the string, and reaches maximum at the string center. Then, irradiance normalization is performed to estimate the fraction of laser power directed at the $\mathrm{NP}(\mathrm{s})$ contributing to the frequency shift, in accordance with the fitted laser beam intensity profile (Figure $2 \mathrm{H}$ ). This step takes into account variations in the focus of the readout laser, which in turn change the local irradiance at the nanoscale. Finally, the experimental absorption cross sections of the probed NPs are obtained by $\mathrm{P}_{\mathrm{abs}} / \mathrm{P}_{\text {total }} \cdot \mathrm{S}$, where $\mathrm{P}_{\mathrm{abs}}$ is the laser power absorbed by the $N P(s), P_{\text {total }}$ is the excitation laser power, and $S$ is the area of the laser spot.

FEM Simulation: $3 \mathrm{D}$ FEM simulations were performed using COMSOL MULTIPHYSICS version 5.4. The EM field distribution was calculated by solving the full field. A domain-backed port, located at the top of the solution domain, was used to excite the linearly polarized incident EM field. A perfectly matched layer (PML) was employed to eliminate nonphysical reflections. Scattering boundary conditions were employed on the top and bottom boundaries of the PML layer to further reduce reflection. For the side planes of the solution domain, Floquet periodicities were used. The geometry of the NPs was modelled according to SEM images. The SiN string was modelled as a substrate below the NPs. Materials were modeled by their complex refractive indexes as functions of wavelength. $34-36$ The structures were surrounded by vacuum. The absorption cross section, i.e., plasmonic heating, is calculated by

$$
\sigma_{a b s}=\frac{1}{I_{0}} \iiint Q d V
$$

where $I_{\mathrm{o}}$ is the intensity of the incident laser, and $Q$ is the power loss density of the NP(s).

\section{RESULTS AND DISCUSSION}

We start from probing the heating of NPs on string resonators and then use the data analysis model detailed in the data analysis section to translate the heating profiles to optical absorption cross sections. Note that when probing plasmonic NPs on a string, the measured relative frequency shift $\delta f$ could also be due to direct laser heating of the string, or vibrational couplings between the NPs and the string. ${ }^{37}$ To clarify their contributions to the relative frequency shift, the following two experiments are first performed:
(I) The frequency shifts of a bare string, heated by a laser with powers to be used for probing the plasmonic NPs, are measured. Results are shown in Figure $\mathrm{S}_{1}$ in the supplementary. It can be seen from Figure $S_{1}$ that the relative frequency shift of the string increases from $\sim 3$ to $\sim 13 \mathrm{ppm}$ when increasing laser power from 40 to $140 \mu \mathrm{W}$. These frequency shifts are significantly lower than those measured on plasmonic NPs placed on strings, shown later in the paper. Specifically, the direct laser heating of the string accounts for only $<3 \%$ of the total relative frequency shift. Therefore, it can be concluded that the contribution of direct laser heating of the string to the total frequency shift is negligible.

(II) Vibration frequencies of a blank quartz fragment, and of the same fragment covered by an AuNS dimer, are measured. For the latter, the probing laser is focused on the center of the dimer. Results are shown in Figure S2 in the supplementary. Figure $\mathrm{S}_{2}$ shows that the relative frequency shift is only 9.7 ppm when probing the AuNS dimer on the quartz fragment. This shift could be due to the vibrational coupling between the AuNS dimer and the quartz. ${ }^{37}$ On a string, under the same experimental condition, the measured relative frequency shift is $\sim 700 \mathrm{ppm}$ (shown in the next section), which is considerably higher. Note that the coupling effect could be enhanced on a string resonator. However, a good agreement between the simulated and the experimentally obtained absorption cross sections (shown later in section 'Optical absorptions of probed NPs') suggests that the major contribution to the probed relative frequency shifts is plasmonic heating.

Probing local heating of AuNS(s): We start with a simple plasmonic system - a single AuNS. A single AuNS shown in Figure $3 \mathrm{~A}$ (left) is irradiated by a $633 \mathrm{~nm}$ HeNe laser in two orthogonal polarizations, and at four different laser powers (40 $\mu \mathrm{W}, 60 \mu \mathrm{W}, 106 \mu \mathrm{W}$ and $140 \mu \mathrm{W}$ ) for each polarization. Note that the laser is partially polarized with an intensity ratio of 4.2:1. This also applies to all subsequent experiments in the following sections. Figure $3^{B}$ shows the relative frequency shift $\delta f$ and the corresponding maximum temperature increase $\Delta T_{\max }$ (calculated using equation 4 ) versus laser power. The inset in Figure $3 \mathrm{~B}$ shows a heatmap generated from the Gaussian fitting (Figure $2 \mathrm{~F}$ ) of the resonance frequency data obtained during probing. It can be seen from Figure ${ }_{3} \mathrm{~B}$ that both $\delta f$ and $\Delta T_{\max }$ vary linearly with incident laser power. This is in accordance with the linear relationship between $\Delta T$ and absorbed laser power $P$ for small temperature changes, given by the equation 33

$$
\Delta T=\frac{1 L}{4 \kappa A} \mathrm{P}
$$

Ideally, for a single AuNS, the heating should be the same in both polarizations. However, Figure $3 \mathrm{~B}$ shows a slight difference. By eliminating the effect of the partially 
polarized intensity ratio (4.2:1) of the laser used in the experiment, and after normalizing irradiance to take into account only the fraction of laser power directed at the NP that leads to the frequency shift (see section 'Data analysis'), a polarization-dependent heating ratio of 1.1 is obtained. This can be attributed to the slight asymmetric geometry of the probed single AuNS. Note that for the rest of the paper, all the experimental heating ratios are obtained using this method.
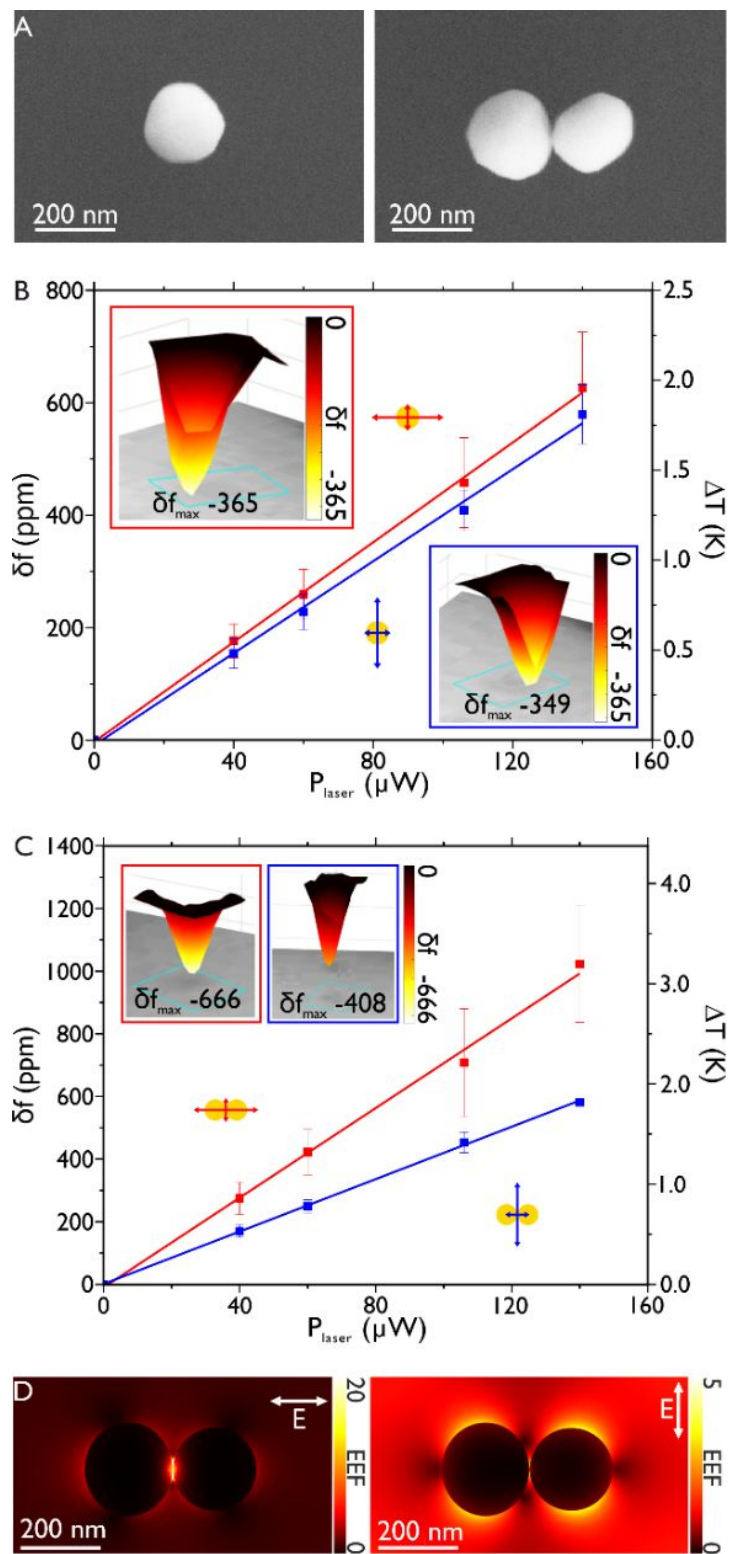

Figure 3. (A) SEM images of the probed AuNS monomer and dimer. $(B, C)$ The relative frequency shift $\delta f$ and the corresponding maximum temperature increase $\Delta \mathrm{T}_{\max }$ versus incident laser power under two orthogonal polarizations for (B) a single AuNS and (C) an AuNS dimer. The insets show representative photothermal images depicting the decrease in resonance frequency on the $\mathrm{SiN}$ strings under both polarizations. (D) Simulated EEF across the center plane of an
AuNS dimer on SiN under the two polarizations at $633 \mathrm{~nm}$ excitation.

Next, a more complex plasmonic system is probed - an AuNS dimer, which is important for applications such as trace molecule detection using SERS38,39 and photothermal plasmon-assisted cancer surgery. ${ }^{9}$ A single AuNS dimer shown in Figure $3 \mathrm{~A}$ (right) is probed using the same partially polarized $633 \mathrm{~nm}$ HeNe laser in two orthogonally different polarizations - along and perpendicular to the dimer axis, and using four incident laser powers. Results are shown in Figure $3 \mathrm{C}$. Similar to the case of the single AuNS, a clear linear relationship between laser power and the temperature induced relative frequency shift is observed. In addition, a difference in the heating efficiency for each polarization direction is observed. Particularly, the relative frequency shift and consequently the temperature increase are higher when the laser is polarized along the axis of the dimer, compared to the case when the laser is polarized perpendicularly to the axis of the dimer.

To investigate further, $3 \mathrm{D}$ FEM simulations are performed. Results are shown in Figure $3 \mathrm{D}$. The left side of Figure $3 \mathrm{D}$ shows the electrical field enhancement factor (EEF) when the laser is polarized along the axis of the dimer, whereas the right side shows the EEF in the case when the laser is polarized perpendicularly. The significant increase of EEF when the polarization is along the axis of the dimer points to stronger LSPR coupling through the nanogap between the two AuNSs. This also means that there is increased plasmonic heating due to stronger absorption of incident laser in this polarization. To quantify, a polarizationdependent heating ratio is calculated to be 2.7 for the simulated model. An experimental polarization-dependent heating ratio of 2.3 is obtained. The good agreement between heating ratios obtained experimentally and by simulation indicate the validity of the experimental method to probe plasmonic heating. The minor difference between experiment and simulation can be attributed to the following: (i) in the simulation, the geometry of the AuNS is assumed to be perfectly symmetrical with a smooth surface. However, as the SEM image in Figure $3 \mathrm{~A}$ (right) shows, the dimer probed in the experiment are asymmetric with a rough surface. Identical and perfectly spherical AuNSs should result in higher heating ratio due to a more predictable difference in the LSPR coupling behavior for the two orthogonal incident polarizations. $4^{\circ}$ (ii) A nanogap of $2 \mathrm{~nm}$ is assumed for the purpose of simulation, however, it is very difficult to determine the actual size of the nanogap from the SEM image. (iii) The simulation considers the two polarization directions to be perfectly orthogonal. In the experiment, this might not exactly be the case since manually controlling rotation of the string (and with it, the probed particles) with respect to the polarization of the incident laser could introduce error. (iv) The roughness of the string may influence the interference pattern of the laser near the probed NPs. In 
the simulation, the surface of the string is assumed to be smooth. Note that for the dimer case, part of the polarization-dependent heating difference should come from the shape anisotropy. However, since the observed difference in polarization-dependent heating of the dimer is much larger than that of the monomer, the different optical absorptions caused by the two polarizationdependent plasmon modes are expected to be the major contributors to the difference in heating.

Probing local heating of shell-isolated Au NPs: Shellisolated $\mathrm{Au}$ NPs have recently gained much interest because of their high detection sensitivity and their applications in plasmon-assisted nanochemistry, photothermal cancer therapy and SERS.7,30 Following the same experimental procedure as detailed in the previous section, a single shell-isolated Au NP, as shown in Figure $4 \mathrm{~A}$ (left), is first probed. Results are shown in Figure $4 \mathrm{~B}$. As expected, a linear relationship is observed.
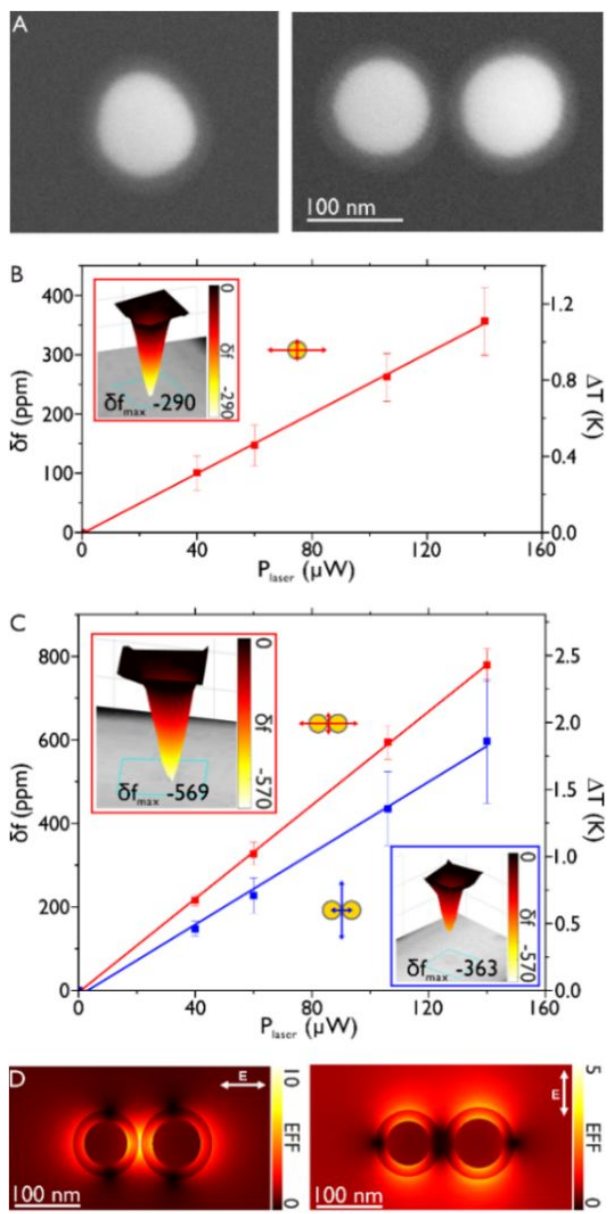

Figure 4. (A) SEM images of the probed single shell-isolated $\mathrm{Au} N \mathrm{NP}$ and its dimer. (B, C) The relative frequency shift $\delta \mathrm{f}$ and the corresponding maximum temperature increase $\Delta \mathrm{T}_{\max }$ versus incident laser power for (B) single and (C) dimer of shell-isolated $\mathrm{Au}$ NPs under two orthogonal polarizations. The insets show representative photothermal images depicting the decrease in resonance frequency on the SiN strings under both polarizations. (D) Simulated EEF across the center plane of a shell-isolated Au NP dimer on SiN under the two polarizations at $633 \mathrm{~nm}$ excitation.

Next, we probe polarization-dependent photothermal heating of a dimer of shell-isolated Au NPs (Figure $4 \mathrm{~A}$, right). Results are shown in Figure ${ }_{4} \mathrm{C}$. In particular, the heating is higher when the polarization direction is along the axis of the dimer. This is similar to the case of an AuNS dimer. Simulation results in Figure $4 \mathrm{D}$ show that the maximum EEF is higher when the laser polarization is along the dimer axis than when it is perpendicular to the dimer axis. This suggests stronger LSPR coupling in the former case, and as a consequence, stronger plasmonic heating. The experimental and simulated polarizationdependent plasmonic heating ratios are calculated to be 1.8 and 2.2, respectively. The difference in the two ratios can be attributed to the following: (i) The presence of the silica shell in the experiment introduces additional thermal mass. This would lead to a reduced temperature contrast on the SiN string for the two polarizations. (ii) Points (iii) and (iv) discussed in the previous section. Additionally, the heating ratio between the two polarizations for the dimer of shell-isolated Au NPs is comparatively lower than that for the AuNS dimers. This can be mainly attributed to the presence of the silica shell, which creates a larger nanogap $(\sim 30 \mathrm{~nm})$ that reduces the strength of LSPR coupling.

Probing local heating of Au nanostars: Au nanostars are another interesting set of particles that are widely used for various applications due to the lightning rod effect of the tips which generate nanoscale EM hot spots in their very close vicinities. ${ }^{4-43}$ To investigate plasmonic heating of $\mathrm{Au}$ nanostars, a single Au nanostar, shown in Figure $5 \mathrm{~A}$ (left), is probed first. Figure ${ }_{5} \mathrm{~B}$ shows the linear response of $\delta f$ and $\Delta T_{\max }$ with increased laser power. The plot shows slightly different heating in the two polarizations. However, after normalization by taking into account the fraction of laser power that leads to the frequency shifts, the polarizationdependent heating ratio becomes $\sim$ 1.o. The plasmon resonances of a nanostar result from the hybridization of plasmons associated with the core and the individual tips. Although the shape anisotropy is large for a nanostar, its plasmon resonance wavelengths are not sensitive to polarization. ${ }^{44}$ In addition, the strengths of its resonances are associated with the size of the tips and the polarization. ${ }^{44}$ In our case, since the tips of the probed nanostar have similar sizes, plus that the excitation wavelength is away from the plasmon resonance wavelength, an observed polarization-dependent heating ratio of $\sim 1.0$ is reasonable. Next, a dimer of Au nanostars (Figure $5 \mathrm{~A}$, right) is probed under two orthogonal polarizations. Results are shown in Figure ${ }_{5} \mathrm{C}$. Although the heating in both polarization directions looks very similar in Figure ${ }_{5} \mathrm{C}$, after normalization, the polarization-dependent heating ratio becomes 1.2. This value is much smaller 
compared to both the AuNS dimer and the shell-isolated $\mathrm{Au}$ NP dimer cases. This could be caused by (i) larger gap distance between the two Au nanostars, (ii) much stronger LSPR at the tips due to the lightning rod effect than that generated via the couplings through the nanogap, ${ }^{45}$ and (iii) large deviation of the excitation wavelength from the LSPR wavelength for the nanostar case.

Probing local heating of AgNCs: The last set of particles probed is the AgNC. In the past decade, nanocube or nanocage structures have been receiving much attention due to their applications in metal-enhanced fluorescence in biological systems, ${ }^{46}$ photothermal destruction of cancer ${ }^{47,48}$ and drug delivery. ${ }^{49}$ We begin with a single $\mathrm{AgNC}$ shown in Figure 6A (left). The single AgNC is probed at four laser powers along two polarization directions, i.e., along the sides and along the diagonal line of the AgNC. Figure $6 \mathrm{~B}$ plots $\delta f$ and $\Delta T_{\max }$ for both polarizations with respect to laser power. A polarization-dependent heating
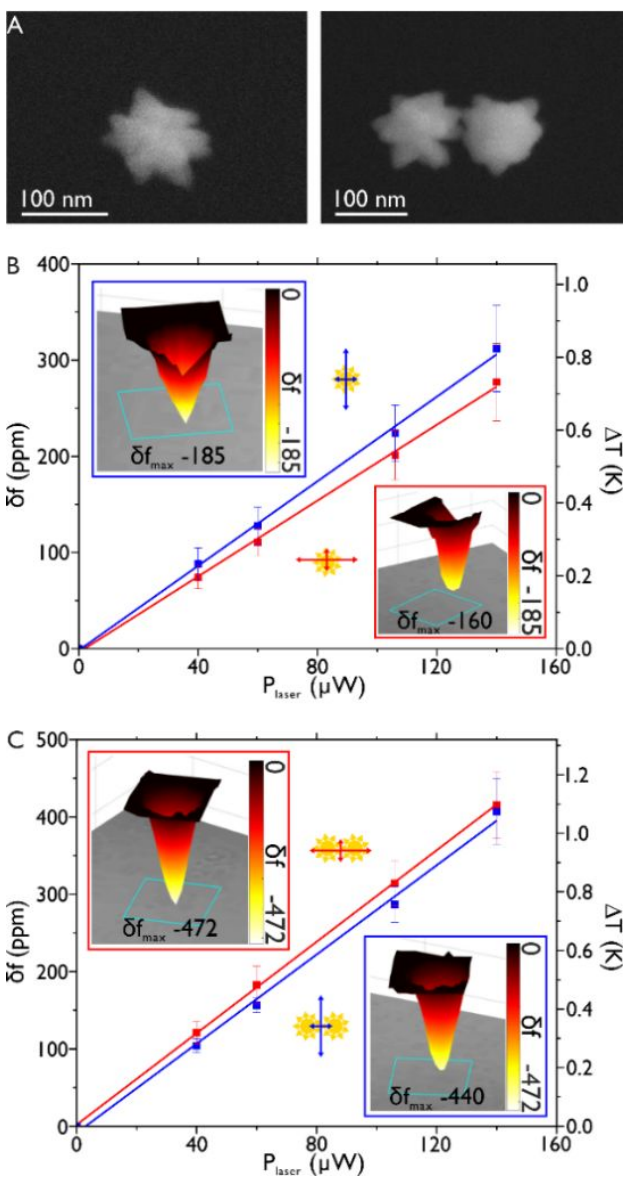

Figure 5. (A) SEM images of the probed $A u$ nanostar monomer and dimer. $(B, C)$ The relative frequency shift $\delta f$ and the corresponding maximum temperature increase $\Delta \mathrm{T}_{\max }$ versus incident laser power under two orthogonal polarizations for (B) a single $\mathrm{Au}$ nanostar and (C) an $\mathrm{Au}$ nanostar dimer. The insets show representative photothermal images depicting the decrease in resonance frequency on the SiN strings under the two polarizations.

ratio of 1.2 is obtained. Note that the simulation of a perfect single AgNC (Figure 6C) shows a polarization-dependent heating ratio of 1.o. This is expected, since the diagonal LSPR mode can be regarded as the superposition of two symmetric LSPR modes oscillating along the sides of the AgNC. The difference in the ratios between the experiment and the simulation can be ascribed to (i) the different shape and sharpness of the corners for the experimentally probed $\mathrm{AgNC}$, and (ii) inaccuracy in change of polarization direction in the experiment, due to manual alignment. A dimer comprising of two closely spaced single AgNCs is probed next. The dimer is shown in Figure 6A (right).
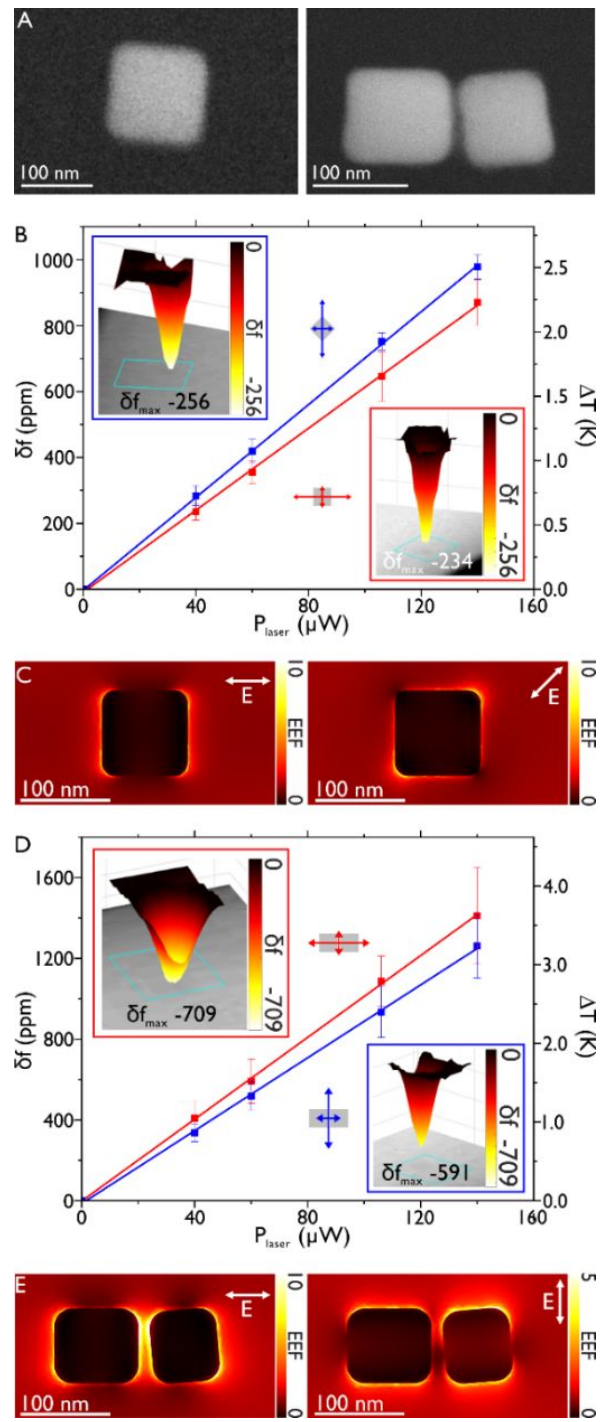

Figure 6. (A) SEM images of the probed AgNC monomer and dimer. (B) The relative frequency shift $\delta f$ and the corresponding maximum temperature increase $\Delta \mathrm{T}_{\max }$ versus incident laser power under two polarizations for a single AgNC. (C) Simulated EEF across the center plane of an AgNC on SiN under two polarizations at $633 \mathrm{~nm}$ excitation. (D) The 
relative frequency shift $\delta \mathrm{f}$ and the corresponding maximum temperature increase $\Delta \mathrm{T}_{\max }$ versus incident laser power under two polarizations for an AgNC dimer. (E) Simulated EEF across the center plane of an AgNC dimer on SiN under two polarizations at $633 \mathrm{~nm}$ excitation. The insets in (B) and (D) show representative photothermal maps depicting the decrease in resonance frequency on the SiN strings under different polarizations.

Results are shown in Figure 6D. It can be seen that $\delta f$ and $\Delta T_{\max }$ vary linearly with laser power. In addition, the AgNC dimer generates more heat when the polarization of the laser is along its axis compared to the perpendicular case. The simulated EEF is plotted in Figure 6E. It shows that the LSPR is stronger when the polarization is along the dimer axis, resulting in more heating. The polarizationdependent heating ratio obtained experimentally is 1.3 whereas the simulated value is 1.2. The slight difference in ratios can be attributed to (i) error introduced by manual alignment of laser polarization with respect to the AgNC dimer axis, (ii) different shape and sharpness for the corners of the probed AgNC dimer, and (iii) size of the nanogap being different in the simulation and in the experiment due to difficulty in its determination from the SEM image.

Optical absorptions of probed NPs: The experimentally quantified and the simulated absorption cross sections of the different types of NPs probed are plotted in Figure 7. The good agreement between the experiments and the simulations indicates the validity of the experimental method and the developed data analysis model to measure optical absorption cross sections of single NP and NP dimers. The slight difference in the absorption cross sections between the experiments and the simulations could be due to (i) surface roughness and shape deformation of the experimentally probed NPs, (ii) roughness of the string, which may influence the interference pattern of the laser near the probed NPs, (iii) errors in the simulation, since the exact sizes of the nanogaps are difficult to determine from the SEM images, and (iv) experimental errors, such as polarization alignment and focus of the laser. In addition, absorption spectra of monomers and dimers of Au NS and shellisolated $\mathrm{Au} \mathrm{NPs}$ on $\mathrm{SiN}$ are simulated and plotted in Figure $\mathrm{S}_{3}$ in the supplementary. It can be seen from Figure $\mathrm{S}_{3}$ that the SiN substrate causes strong red shifts to plasmon resonances of Au spherical nanoparticles due to its high refractive index. Also, the wavelength of excitation (633 $\mathrm{nm}$ ) is far from the plasmon resonance wavelengths, for all the cases. This reveals that the method can be used for quantification of optical absorption cross sections of individual plasmonic structures whose LSPR wavelengths are far from that of the excitation wavelength. This is attributed to the excellent sensitivity of the method ( $~ 89$ $\mathrm{Hz} / \mathrm{K})$.

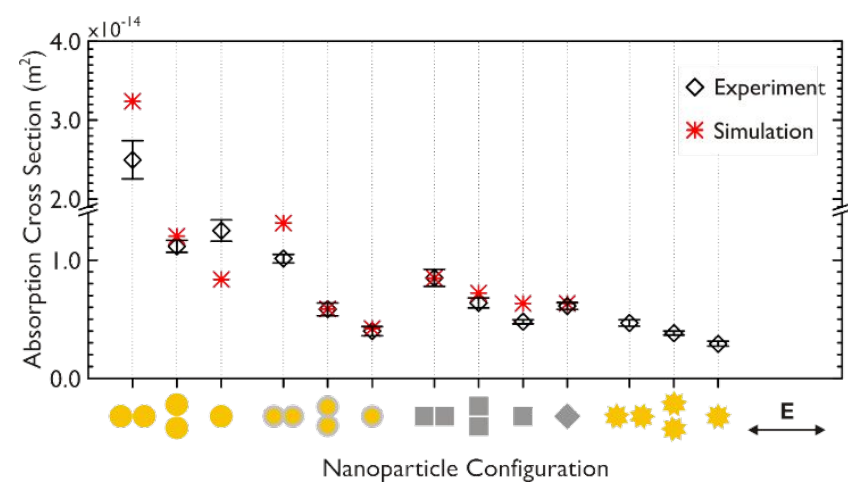

Figure 7. The experimental and the simulated absorption cross sections of the different types of NPs on SiN string resonators under different polarization directions of excitation.

\section{CONCLUSION}

In conclusion, we have demonstrated the use of $\mathrm{SiN}$ micromechanical string resonators as an excellent tool for profiling optical absorption cross sections of individual plasmonic NPs and their dimers, even of those consisting of extremely small nanogaps or exhibiting LSPR wavelengths far from the excitation. Our results shed light on the thermoplasmonic behavior of the probed NPs and illustrate that the plasmonic heating is strongly influenced by the actual particle geometry and morphology. Moreover, the developed method and the obtained optical absorption profiles of the different NPs with different configurations facilitate future development and optimization of thermoplasmonic applications. Our work not only facilitates a better fundamental understanding of thermoplasmonics, but also opens up new avenues for the application of these nano-sources of heat.

\section{ASSOCIATED CONTENT}

Supporting Information. Experimental results demonstrating the (i) relative frequency shift of a bare string irradiated by a $633 \mathrm{~nm}$ laser and (ii) relative frequency shifts of a blank quartz fragment and the same piece but covered with an AuNS dimer.

This material is available free of charge via the Internet at http://pubs.acs.org

\section{AUTHOR INFORMATION}

\section{Corresponding Author}

*Kaiyu Wu (Email: kaiwu@dtu.dk)

\section{Present Addresses}

† Ibsen Photonics A/S, Ryttermarken 17, 3520 Farum, Denmark 
†† Radiometer Medical ApS, Åkandevej 21, 2700 Brønshøj, Denmark

\section{Author Contributions}

The manuscript was written through contributions of all authors

\section{Funding Sources}

This work was supported by the Danish National Research Foundation (Project DNRF122) and Villum Fonden (Grant No. 9301).

\section{Notes}

The authors declare no competing financial interest.

\section{ACKNOWLEDGMENT}

The authors would like thank Dr. Chiara Mazzoni for her help on SEM characterizations and Dr. Peter Ouma Okeyo for his help in preparing the quartz sample. The authors would also like to thank the cleanroom staff at the National Centre for Nano-Fabrication and Characterization at the Technical University of Denmark (DTU Nanolab), especially process specialist Dr. Karen Birkelund, for her extensive guidance. 


\section{REFERENCES}

(1) Boriskina, S. V.; Ghasemi, H.; Chen, G. Plasmonic Materials for Energy: From Physics to Applications. Mater. Today 2013, 16 (10), 375386.

(2) De Sio, L.; Placido, T.; Comparelli, R.; Lucia Curri, M.; Striccoli, M.; Tabiryan, N.; Bunning, T. J. Next-Generation Thermo-Plasmonic Technologies and Plasmonic Nanoparticles in Optoelectronics. Prog. Quantum Electron. 2015, 41, 23-70.

(3) Chen, Y.; Ming, H. Review of Surface Plasmon Resonance and Localized Surface Plasmon Resonance Sensor. Photonic Sensors 2012, 2 (1), 37-49.

(4) Govorov, A. O.; Richardson, H. H. Generating Heat with Metal Nanoparticles. Nano Today 2007, 2 (1), 30-38.

(5) Jain, P. K.; Huang, X.; El-Sayed, I. H.; El-Sayed, M. A. Noble Metals on the Nanoscale: Optical and Photothermal Properties and Some Applications in Imaging, Sensing, Biology, and Medicine. Acc. Chem. Res. 2008, 41 (12), 15781586.

(6) Huang, X.; Jain, P. K.; El-Sayed, I. H.; El-Sayed, M. A. Plasmonic Photothermal Therapy (PPTT) Using Gold Nanoparticles. Lasers Med. Sci. 2008, 23 (3), 217-228.

(11) Alkilany, A. M.; Thompson, L. B.; Boulos, S. P.; Sisco, P. N.; Murphy, C. J. Gold Nanorods: Their Potential for Photothermal Therapeutics and Drug Delivery, Tempered by the Complexity of Their Biological Interactions. Adv. Drug Deliv. Rev. 2012, 64 (2), 190-199.

(12) Sershen, S. R.; Westcott, S. L.; Halas, N. J.; West, J. L. Temperature Sensitive PolymerNanoshell Composites for Photothermally Modulated Drug Delivery. J. Biomed. Mater. Res. 2000, 51 (3), 293-298.

(13) Kang, B.; Afifi, M. M.; Austin, L. A.; El-Sayed, M. A. Exploiting the Nanoparticle Plasmon Effect: Observing Drug Delivery Dynamics in Single Cells via Raman/Fluorescence Imaging Spectroscopy. ACS Nano 2013, 7 (8), 7420-7427.

(14) Boyer, D.; Tamarat, P.; Maali, A.; Lounis, B.; Orrit, M. Photothermal Imaging of NanometerSized Metal Particles Among Scatterers. Science (80-. ). 2002, 297 (5584), 1160-1163.

(15) Pache, C.; Bocchio, N. L.; Bouwens, A.; Villiger, M.; Berclaz, C.; Goulley, J.; Gibson, M. I.; Santschi, C.; Lasser, T. Fast Three-Dimensional Imaging of Gold Nanoparticles in Living Cells with Photothermal Optical Lock-in Optical Coherence Microscopy. Opt. Express 2012, 20 (19), 21385.

(16) Donner, J. S.; Morales-Dalmau, J.; Alda, I.; Marty, R.; Quidant, R. Fast and Transparent Adaptive Lens Based on Plasmonic Heating. ACS Photonics 2015, 2 (3), 355-360.

(17) Boyd, D. A.; Greengard, L.; Brongersma, M.; ElNaggar, M. Y.; Goodwin, D. G. PlasmonAssisted Chemical Vapor Deposition. Nano Lett. 2006, 6 (11), 2592-2597.

(18) Thomson Reuters, Web of Science Core Collection.

(9) Lukianova-Hleb, E. Y.; Kim, Y. S.; Belatsarkouski, I.; Gillenwater, A. M.; O’Neill, B. E.; Lapotko, D. O. Intraoperative Diagnostics and Elimination of Residual Microtumours with Plasmonic Nanobubbles. Nat. Nanotechnol. 2016, 11 (6), 525-532.

(10) Khlebtsov, N. G.; Dykman, L. A. Optical Properties and Biomedical Applications of Plasmonic Nanoparticles. J. Quant. Spectrosc. Radiat. Transf. 2010, 111 (1), 1-35.

(19) Majumdar, A. Scanning Thermal Microscopy. Annu. Rev. Mater. Sci. 1999, 29 (1), 505-585.

(20) Baffou, G.; Bon, P.; Savatier, J.; Polleux, J.; Zhu, M.; Merlin, M.; Rigneault, H.; Monneret, S. Thermal Imaging of Nanostructures by Quantitative Optical Phase Analysis. ACS Nano 2012, 6 (3), 2452-2458.

(21) Hu, S.; Liu, B.-J.; Feng, J.-M.; Zong, C.; Lin, K.Q.; Wang, X.; Wu, D.-Y.; Ren, B. Quantifying Surface Temperature of Thermoplasmonic 
Nanostructures. J. Am. Chem. Soc. 2018, 140 (42), 13680-13686.

(22) Carlson, M. T.; Khan, A.; Richardson, H. H. Local Temperature Determination of Optically Excited Nanoparticles and Nanodots. Nano Lett. 2011, 11 (3), 1061-1069.

(23) Baffou, G.; Kreuzer, M. P.; Kulzer, F.; Quidant, R. Temperature Mapping Near Plasmonic Nanostructures Using Fluorescence Polarization Anisotropy. Opt. Express 2009, 17 (5), 3291.

(24) Mann, S. A.; Sciacca, B.; Zhang, Y.; Wang, J.; Kontoleta, E.; Liu, H.; Garnett, E. C. Integrating Sphere Microscopy for Direct Absorption Measurements of Single Nanostructures. ACS Nano 2017, 11 (2), 1412-1418.

(25) Verbridge, S. S.; Craighead, H. G.; Parpia, J. M. A Megahertz Nanomechanical Resonator with Room Temperature Quality Factor Over a Million. Appl. Phys. Lett. 2008, 92 (1), 013112.

(26) Larsen, T.; Schmid, S.; Grönberg, L.; Niskanen, A. O.; Hassel, J.; Dohn, S.; Boisen, A. Ultrasensitive String-Based Temperature Sensors. Appl. Phys. Lett. 2011, 98 (12), 121901.

(27) Larsen, T.; Schmid, S.; Boisen, A. Micro String Resonators as Temperature Sensors. AIP Conf. Proc. 2013, 15528 (2013), 931-936.

(28) Schmid, S.; Wu, K.; Larsen, P. E.; Rindzevicius, T.; Boisen, A. Low-Power Photothermal Probing of Single Plasmonic Nanostructures with Nanomechanical String Resonators. Nano Lett. 2014, 14 (5), 2318-2321.

(29) Jauffred, L.; Samadi, A.; Klingberg, H.; Bendix, P. M.; Oddershede, L. B. Plasmonic Heating of Nanostructures. Chem. Rev. 2019, 119 (13), 80878130.

(30) Li, J. F.; Huang, Y. F.; Ding, Y.; Yang, Z. L.; Li, S. B.; Zhou, X. S.; Fan, F. R.; Zhang, W.; Zhou, Z. Y.; Wu, D. Y.; et al. Shell-Isolated NanoparticleEnhanced Raman Spectroscopy. Nature 2010, 464 (7287), 392-395.

(31) Schmid, S.; Jensen, K. D.; Nielsen, K. H.; Boisen, A. Damping Mechanisms in High-Q Micro and Nanomechanical String Resonators. Phys. Rev. B - Condens. Matter Mater. Phys. 2011, 84 (16), 1-6.

(32) Larsen, T.; Schmid, S.; Villanueva, L. G.; Boisen, A. Photothermal Analysis of Individual Nanoparticulate Samples Using Micromechanical Resonators. ACS Nano 2013, 7 (7), 6188-6193.
（33） Schmid, S.; Villanueva, L. G.; Roukes, M. L. Fundamentals of Nanomechanical Resonators. Springer International Publishing. Cham 2016.

(34) Johnson, P. B.; Christy, R. W. Optical Constants of the Noble Metals. Phys. Rev. B 1972, 6 (12), 4370-4379.

(35) Philipp, H. R. Optical Properties of Silicon Nitride. J. Electrochem. Soc. 1973, 120 (2), 295.

(36) Malitson, I. H. Interspecimen Comparison of the Refractive Index of Fused Silica. J. Opt. Soc. Am. 1965, 55 (10), 1205.

(37) Yi, C.; Dongare, P. D.; Su, M.-N.; Wang, W.; Chakraborty, D.; Wen, F.; Chang, W.-S.; Sader, J. E.; Nordlander, P.; Halas, N. J.; et al. Vibrational Coupling in Plasmonic Molecules. Proc. Natl. Acad. Sci. U. S. A. 2017, 114 (44), 11621-11626.

(38) Wu, K.; Nguyen, L. Q.; Rindzevicius, T.; Keller, S. S.; Boisen, A. Wafer-Scale Polymer-Based Transparent Nanocorals with Excellent Nanoplasmonic Photothermal Stability for High-Power and Superfast SERS Imaging. Adv. Opt. Mater. 2019, 1901413.

（39） Qian, X.-M.; Nie, S. M. Single-Molecule and Single-Nanoparticle SERS: From Fundamental Mechanisms to Biomedical Applications. Chem. Soc. Rev. 2008, 37 (5), 912.

(40) Yoon, J. H.; Selbach, F.; Schumacher, L.; Jose, J.; Schlücker, S. Surface Plasmon Coupling in Dimers of Gold Nanoparticles: Experiment and Theory for Ideal (Spherical) and Nonideal (Faceted) Building Blocks. ACS Photonics 2019, 6 (3), 642-648.

(41) Vo-Dinh, T.; Liu, Y.; Crawford, B. M.; Wang, H.N.; Yuan, H.; Register, J. K.; Khoury, C. G. Shining Gold Nanostars: From Cancer Diagnostics to Photothermal Treatment and Immunotherapy. J Immunol Sci. 2018, 2 (1), 1-8

(42) Song, C.; Li, F.; Guo, X.; Chen, W.; Dong, C.; Zhang, J.; Zhang, J.; Wang, L. Gold Nanostars for Cancer Cell-Targeted SERS-Imaging and NIR Light-Triggered Plasmonic Photothermal Therapy (PPTT) in the First and Second Biological Windows. J. Mater. Chem. B 2019, 7 (12), 2001-2008.

(43) Khoury, C. G.; Vo-Dinh, T. Gold Nanostars For Surface-Enhanced Raman Scattering: Synthesis, Characterization and Optimization. J. Phys. Chem. C 2008, 112 (48), 18849-18859.

(44) Hao, F.; Nehl, C. L.; Hafner, J. H.; Nordlander, 
P. Plasmon Resonances of a Gold Nanostar.

Nano Lett. 2007, 7 (3), 729-732.

(45) Wu, K.-Y.; Cheng, X.-L.; Lee, L. P. Intra-Particle Coupling and Plasmon Tuning of Multilayer $\mathrm{Au} /$ Dielectric/Au Nanocrescents Adhered to a Dielectric Cylinder. Nanotechnology 2012, 23 (5), 055201.

(46) Camposeo, A.; Persano, L.; Manco, R.; Wang, Y.; Del Carro, P.; Zhang, C.; Li, Z.-Y.; Pisignano, D.; Xia, Y. Metal-Enhanced Near-Infrared Fluorescence by Micropatterned Gold Nanocages. ACS Nano 2015, 9 (10), 10047-10054.

(47) Chen, J.; Wang, D.; Xi, J.; Au, L.; Siekkinen, A.; Warsen, A.; Li, Z. Y.; Zhang, H.; Xia, Y.; Li, X. Immuno Gold Nanocages With Tailored Optical Properties for Targeted Photothermal Destruction of Cancer Cells. Nano Lett. 2007, 7 (5), 1318-1322.

(48) Xia, Y.; Li, W.; Cobley, C. M.; Chen, J.; Xia, X.; Zhang, Q.; Yang, M.; Cho, E. C.; Brown, P. K. Gold Nanocages: From Synthesis to Theranostic Applications. Acc. Chem. Res. 2011, 44 (10), 914924 .

(49) Cobley, C. M.; Au, L.; Chen, J.; Xia, Y. Targeting Gold Nanocages to Cancer Cells for Photothermal Destruction and Drug Delivery. Expert Opin. Drug Deliv. 2010, 7 (5), 577-587. 


\section{For TOC only}
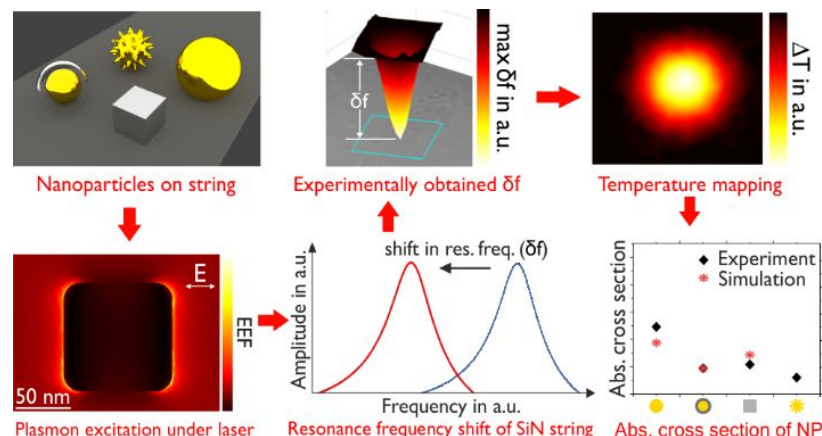